\title{
Utilização do Índice de conicidade no diagrama de manejo de densidade em povoamentos de Pinus taeda L.
}

\author{
Use of the slenderness index in the stand \\ density management diagram of Pinus taeda $\mathrm{L}$.
}

\author{
Paulo Sérgio Pigatto Schneider1, Solon Jonas Longhi², Paulo Renato Schneider ${ }^{3}$, \\ Evandro Alcir Meyer ${ }^{4}$ e Junior Oliveira Mendes ${ }^{4}$
}

\begin{abstract}
Resumo
O presente trabalho foi desenvolvido com o objetivo de estruturar, com base em diagrama de densidade, a produção volumétrica para populações de Pinus taeda L., observando a variação do coeficiente conicidade com a densidade populacional, o índice de espaçamento relativo e diâmetro médio quadrático. Para isto, foram tomados dados de 128 parcelas permanentes, medidas anualmente até os 18 anos, em experimento instalado em povoamentos implantados em diferentes espaçamentos, manejados em densidade completa e altamente estocados. Os diagramas de manejo da densidade (DMDs) para os povoamentos, elaborados com cruzamento de variáveis dendrométricas: número de árvores por hectare, altura dominante, diâmetro médio e volume por hectare apresentaram uma boa eficiência e baixo erro de estimativa. A introdução do índice de espaçamento relativo e do coeficiente de conicidade no DMD permitiram estabelecer uma zona ótima de manejo da densidade populacional para manter uma maior estabilidade do povoamento contra a ação do vento.
\end{abstract}

Palavras-chave: competição; crescimento; desbaste; produção.

\begin{abstract}
This work was developed with the aim of structuring the volumetric production based on density management diagram for populations of Pinus taeda L., noting the slenderness coefficient variation with population density, relative spacing index and diameter of basal area. For this, data from 128 permanent plots were taken, measured annually until age 18, in an experiment established within stands implanted at various spacing, managed in complete and highly stocked density. The stands density management diagrams (DMDs) made by cross-comparing dendrometric variables: number of trees per hectare; dominant height; diameter and volume per hectare; all showed a good efficiency with low error in the estimate. The introduction of the relative spacing index and the coefficient of slenderness in DMD allowed us to establish a great area of management of population density to maintain greater stand stability of against the wind.
\end{abstract}

Keywords: competition; growth; thinning; yield.

\section{INTRODUÇÃO}

A determinação dos níveis adequados de estoque de crescimento é um processo complexo que envolve fatores biológicos, tecnológicos, econômicos e operacionais específicos numa situação particular de manejo. O processo requer a seleção dos limites superior e inferior de estoque de crescimento. O primeiro é escolhido para se obter crescimento numa posição aceitável, com vigor da árvore individual, enquanto o último é escolhido para manter uma ocupação aceitável do local. Do ponto de vista biológico, os níveis de estoque de crescimento devem ser limitados dentro das densidades suportes correspondentes ao limiar de autodesbaste e fechamento do dossel (DEAN; BALDWIN, 1996). No entanto, a tradução dos objetivos de manejo específicos para os níveis superior e inferior do estoque de crescimento é o passo mais difícil na criação de um regime de manejo da densidade (DAVIS, 1966).

\footnotetext{
${ }^{1}$ Doutor em Engenharia Florestal. UFSM - Universidade Federal de Santa Maria. Av. Roraima, s/n- Camobi - 97105900 Santa Maria, RS, Brasil. E-mail: paulosergiopigatoschneider@gmail.com.

2Professor Sênior do Departamento de Ciências Florestais. UFSM - Universidade Federal de Santa Maria. Av. Roraima, s/nCamobi - 97105900 - Santa Maria, RS, Brasil. E-mail: longhi.solon@gmail.com.

3Professor Titular do Departamento de Ciências Florestais. UFSM - Universidade Federal de Santa Maria. Av. Roraima, s/nCamobi - 97105900 - Santa Maria, RS, Brasil. E-mail: paulorenato@gmail.com.

${ }^{4}$ Doutorando em Engenharia Florestal. UFSM - Universidade Federal de Santa Maria. Av. Roraima, s/n- Camobi - 97105900 - Santa Maria, RS, Brasil. E-mail: eam.meyer@gmail.com.
} 
Apesar dos ensaios de campo serem a melhor maneira de determinar o momento de desbastes e os limites teóricos mencionados acima possuem duas limitações sérias (DEAN; BALDWIN, 1993): levam muitos anos para ser concluídos e os resultados não podem ser aplicados com precisão em locais onde a qualidade do sítio e objetivos de manejo são diferentes dos encontrados nos ensaios. Uma abordagem alternativa é a utilização de diagramas de manejo de densidade do povoamento, que são modelos da média do nível de suporte das relações entre o rendimento e a densidade em todos os estágios de desenvolvimento (NEWTON; WEETMAN, 1994).

Em povoamentos florestais, a mortalidade das árvores pode ocorrer de forma regular ou irregular. A mortalidade regular está ligada a fatores exógenos e danos causados pelo homem (LEE, 1971), bem como a competição entre as árvores. A mortalidade irregular é definida como morte casual, ligada a fatores endógenos, definidos pelo espaço e tempo, podendo manifestar-se a partir do plantio, em consequência de mudas menos vigorosas (BURKHART, 1974).

Para estudar o autodesbaste, Yoda et al. (1963) formularam a lei da potência de valor -3/2, que descreve a proporção máxima do número médio de árvores pelo tamanho, para uma determinada espécie, em que a mortalidade é dependente da densidade. A razão máxima entre o número de árvores e seu tamanho tem idêntica taxa de crescimento para todos os locais, e o autodesbaste deve ocorrer quando as árvores ocuparem completamente o sítio. Essa lei da potência -3/2 é representada na equação por: $\ln (\mathrm{W})=\ln (\mathrm{a})-(3 / 2) \cdot \ln (\mathrm{N})$, em que, $\mathrm{N}$ é o número de árvores por hectare, W é a variável dependente e "a" é uma constante de proporcionalidade. Tang et al. (1994) estenderam a teoria do autodesbaste para povoamentos em densidade completa e subestocados. No modelo, a lei do autodesbaste pode ser considerada um caso especial, pois permite obter para um diâmetro qualquer a densidade e a produção. Esta lei do autodesbaste tem sido objeto de estudo para uso prático e de discussões acerca da sua eficiência no manejo florestal por vários pesquisadores, como: Westoby (1981), Zeide (1987 e 1991), Smith e Hann (1986), Yoda et al. (1963), Del Rio et al. (2001), entre outros.

Observações casuais mostram que com o aumento da densidade dos povoamentos, o crescimento em diâmetro é reduzido em relação ao crescimento em altura. Neste caso as árvores adquirem forma alongada (menor conicidade), enquanto em povoamentos muito abertos as árvores adquirem robustez (maior conicidade).

Segundo Baker (1950), para determinar a variação da relação da forma de tronco das árvores com a densidade populacional pode-se utilizar o coeficiente slenderness, traduzido como sendo a conicidade por densidade, postulado para medir a densidade de povoamentos equiâneos, através da expressão da forma slenderness das árvores que compõem o povoamento. Este coeficiente slenderness é expresso por: $C S=\left[(t \cdot h)^{b}\right] /[t \cdot d g]$, em que, t é a idade, h a altura média, dg o diâmetro quadrático médio, e b é uma constante, que varia com a densidade do povoamento e espécie. Matematicamente, esta proporção é algo obscuro, mas quando a constante b assume valor equivalente a 1,0 então o t da fórmula é simplificada, passando a ser expressa por: $C S=h / d_{g}$. Essa fórmula mede, em povoamentos de densidade controlada, a relação entre a altura/diâmetro, que normalmente é expressa por uma constante que varia com a densidade, espécie e até certo grau com sua tolerância.

$\mathrm{Na}$ Europa, os silvicultores utilizam o índice slenderness para avaliar a susceptibilidade a danos por neve e vento, que agem sobre a copa das árvores para muitos anos de desenvolvimento (NYKÄNEN et al., 1997). A tendência do relacionamento da razão da altura:diâmetro com a incidência de danos por neve e vento têm sido muito forte (BRUNIG, 1974; FABER, 1975), como exemplo as coníferas do norte da Europa têm demonstrado maior probabilidade de dano por vento, com o aumento da relação da altura:diâmetro (LOHMANDER; HELLES, 1987). No entanto, Cremer et al. (1982), em populações de Pinus radiata na Nova Zelândia, não encontraram nenhum dano por vento, vinculado a relação de altura:diâmetro abaixo de 74, quando ambas as variáveis são medidas em unidades idênticas, e ocorrência de danos por vento quase completo em proporções superiores a 90.

Através da abordagem de correlação de dados das árvores de parcelas permanentes, em Alberta, nos EUA, as relações entre os índices slenderness e variáveis de árvores foram investigadas para cinco principais espécies boreais. Neste caso, os índices slenderness encontrados em árvores correlacionaram-se negativamente com diâmetro, altura, comprimento da copa e faixa etária das árvores, mas positivamente com o nível de densidade suporte, composição de espécies e os valores de índice de sítio. Para três espécies de coníferas, os resultados foram consistentes com aqueles encontrados para 
espécies européias de coníferas. No entanto, para as duas espécies caducifólias, não encontraram estudos semelhantes para comparação. Os resultados indicaram que cerca de $50 \%$ das amostras tiveram um índice slenderness superior a 100, podendo estar em alto risco devido a ação do vento (WANG et al., 1998).

Segundo Efthimiou (2012), o índice slenderness é um fator importante, que descreve o tipo de caule que cada espécie florestal desenvolve, o que depende da própria espécie, da idade e as condições do local. É um fator essencial que caracteriza a estrutura e estabilidade de suporte às árvores e um meio para a avaliação da dinâmica do desenvolvimento da altura. No seu estudo, constatou que espécie de salgueiro encontrados em florestas mistas e puras, em uma área protegida, com uma estrutura de floresta alta, o coeficiente slenderness de Salix fragilis eram inferiores de 40, que indica estrutura de suporte mais estável, em comparação com indivíduos jovens de Salix alba, que atinge valores semelhantes com quase o dobro do diâmetro do fuste.

Pardé e Bouchon (1988) consideraram suportes estáveis das árvores à ação do vento uma posição média de coeficiente slenderness (CS) < 80 e como instável quando CS > 100. Da mesma forma, Hinze e Wessels (2002) relataram que um valor individual de CS > 85 implica em alta instabilidade da árvore devido à ação do vento ou neve. Também, Castedo-Dorado et al. (2000) constataram que a neve e o vento em plantações de Pinus radiata na Espanha, causam danos em povoamentos com CS $>88$, especialmente em áreas montanhosas.

Por outro lado, para Rice e Man (2011), o coeficiente de conicidade ou slenderness médios (CS) para o total de árvores de várias espécies danificadas pelo vento e com avaliação anual, variou entre os tratamentos de desbaste, colheita, espécies e anos de avaliação. Para as espécies de árvores dominantes no povoamento, a maior média do CS foi de 93,8, nas parcelas parcialmente colhidas. Não foi encontrada diferença estatisticamente significativa no CS, entre árvores vivas e as danificadas pelo vento, entre as espécies de árvores ou períodos de avaliação.

Os diagramas de manejo de densidade do povoamento podem ser utilizados para obter programas de controle de densidade para vários objetivos de manejo (NEWTON, 1997): (i) para minimizar a oportunidade temporal para a obtenção de critérios específicos de operacionalidade, (ii) controlar o desenvolvimento das árvores durante os estágios iniciais de desenvolvimento, e (iii) otimizar a densidade para objetivos específicos de qualidade da madeira, vegetação ou de manejo da vida selvagem. O uso de diagramas de manejo de densidade do povoamento é, portanto, um dos métodos mais eficazes disponíveis para a projeção, exibição e avaliação de sistemas de manejo alternativos da densidade em povoamentos equiâneos.

Um modelo de nível padrão foi desenvolvido para determinar o volume de Pinus pinaster Ait. de povoamentos equiâneos, sob uma ampla gama de condições de densidade e sítio. O modelo desenvolvido tem grande potencial de pois os dados necessários para as equações e criação dos diagramas são de fácil obtenção e geralmente estão disponíveis a partir de estoques normais de produção de madeira. As parcelas utilizadas para desenvolver estes diagramas foram estratificadas em uma ampla gama de pesos, portanto, o modelo de nível padrão é de uso adicional, pois tratamentos silviculturais podem induzir mudanças na estrutura ao longo do tempo e isto é levado em conta no modelo. Embora este modelo de nível de suporte foi desenvolvido principalmente para a derivação e momentos de desbastes, para fazer a estimativa rápida de volume, também é útil para outros fins. Assim, se a informação adicional disponível, o diagrama pode ser usado para descrever a dinâmica de povoamentos de várias perspectivas de manejo de recursos (por exemplo, produção de recursos não-madeireiros ou habitat de espécies selvagens) sobrepondo essas informações em um diagrama de manejo da densidade, facilitando as decisões de gestão (BARRIO-ANTA et al., 2005).

Face a isto, o presente trabalho foi desenvolvido com o objetivo de estruturar a produção volumétrica em diagrama de manejo da densidade para populações de Pinus taeda. Para isto, definiram-se como objetivos específicos: estudar a variação do coeficiente de conicidade ou slenderness com a densidade populacional e diâmetro médio; determinar o momento da ocorrência do autodesbaste em relação à variação do coeficiente de conicidade, índice de espaçamento relativo e diâmetro quadrático médio ; elaborar um diagrama de manejo da densidade conciliando as variáveis dendrométricas: produção, diâmetro médio, altura dominante, densidade populacional, coeficiente slenderness e índice de espaçamento relativo. 
Schneider et al. - Utilização do Índice de conicidade no diagrama

de manejo de densidade em povoamentos de Pinus taeda $\mathrm{L}$.

\section{MATERIAL E MÉTODOS}

Os dados foram levantados em áreas pertencentes à Empresa Klabin S.A., na unidade localizada no município de Otacílio Costa, região fisiográfica denominada de Planalto Central Catarinense.

O clima da região, segundo a classificação climática de Köppen, é do tipo "Cfb", mesotérmico, subtropical úmido, com verões frescos, não apresentando estação seca definida. A precipitação média anual é de $1.808 \mathrm{~mm}$, distribuída em 124 dias e temperatura média anual de $17,8^{\circ} \mathrm{C}$ (MOTA et al., 1971). O solo da área do experimento é classificado como Latossolo Bruno alumínico, horizonte A húmico, textura argilosa e relevo suavemente ondulado (EMBRAPA, 1999).

Para esta pesquisa foram utilizados dados de duas fontes. A primeira foi proveniente de um experimento de espaçamento, sendo utilizado dados de povoamentos com espaçamento de 1,5 x 1,0 e $1,5 \times 2,0$, tendo resultado em 6 unidades amostrais permanentes, medidas até os 18 anos. Em cada unidade amostral foram medidas as circunferências à altura do peito, altura das árvores, bem como anotação de danos provocados por vento. Os dados de número de árvores por hectare e diâmetro médio correspondentes são oriundos de parcelas permanentes com espaçamento acima mencionado, tendo originado 50 observações em cada espaçamento. Na segunda fonte de dados, foram tomados um total de 123 parcelas permanentes de dimensão de $20 \mathrm{~m}$ x $30 \mathrm{~m}$, instaladas em povoamentos comerciais, com idade variando entre 6 a 18 anos, medidas periodicamente, manejadas em regime de desbaste por baixo, com densidade controlada pelo índice de espaçamento relativo.

Na Tabela 1 encontram-se sumarizados os dados dendrométricos oriundos das parcelas permanentes utilizados no ajuste dos modelos para elaboração dos diagramas de manejo da densidade.

Tabela 1. Sumário dos dados amostrados das parcelas permanentes utilizadas para desenvolvimento do estudo.

Table 1. Summary data to the sample of permanent plots used for study development.

\begin{tabular}{lcccc}
\hline Variáveis dos povoamentos & Média & Desvio Padrão & Mínimo & Máximo \\
\hline $\mathrm{t}($ anos $)$ & 13,7 & 2,09 & 6,0 & 18,0 \\
$\mathrm{~N}(\mathrm{ha})$ & 1147 & 38,3 & 304 & 1744 \\
$\mathrm{dg}(\mathrm{cm})$ & 22,5 & 3,47 & 12,9 & 33,1 \\
$d(\mathrm{~cm})$ & 22,1 & 3,49 & 12,3 & 32,7 \\
$h(\mathrm{~m})$ & 17,9 & 2,96 & 10,0 & 27,9 \\
$\mathrm{~h} 100(\mathrm{~m})$ & 19,72 & 2,89 & 12,9 & 29,0 \\
$\mathrm{RS}(\%)$ & 15,87 & 2,77 & 12,02 & 23,89 \\
$\mathrm{CS}(\mathrm{m} / \mathrm{cm})$ & 0,81 & 0,04 & 0,75 & 0,93 \\
$\mathrm{~V}\left(\mathrm{~m}^{3} \mathrm{ha}^{-1}\right)$ & 324,07 & 102,19 & 56,0 & 573,0 \\
\hline
\end{tabular}

Em que: $\mathrm{t}=$ idade (anos); $\mathrm{N}=$ número de árvores por hectare; $\mathrm{d}=$ diâmetro quadrático médio $(\mathrm{cm}) ; \mathrm{d}=$ diâmetro médio do povoamento ( $\mathrm{cm}$ ); $h=$ altura média do povoamento $(\mathrm{m}) ; \mathrm{h}_{100}=$ altura dominante $(\mathrm{m}) ; \mathrm{RS}=$ índice de espaçamento relativo $(\%)$; CS = coeficiente de conicidade ou slenderness $(\mathrm{m} / \mathrm{cm}) ; \mathrm{V}=$ volume remanescente total $\left(\mathrm{m}^{3} / \mathrm{ha}^{-1}\right)$.

Os Diagramas de Manejo da Densidade do Povoamento (DMD) desenvolvidos no presente estudo incluíram o índice de espaçamento relativo (RS \%) e um sistema de três equações com componentes básicos, descrito por Castedo-Dorado et al. (2009), baseando-se no trabalho pioneiro de Becking (1954), utilizando diferentes formas para construir um sistema de manejo da densidade, conforme descrito por Jack e Long (1996).

O índice de espaçamento relativo foi utilizado para caracterizar o nível de estoque de crescimento, que foi calculado como a razão da distância média entre as árvores e altura dominante, expresso em percentagem. Assim, assumindo um espaçamento triangular, o índice de espaçamento relativo (RS) foi determinado por:

$$
R S=\frac{\sqrt{20000 /(N \cdot \sqrt{3})}}{h_{100}} \cdot 100
$$

Em que: RS é o índice de espaçamento relativo; $\mathrm{N}$ o número de árvores por hectare, e $\mathrm{h}_{100}$ a altura dominante

Os procedimentos para a construção dos diagramas de manejo da densidade envolveram os seguintes passos, definido por Barrio-Anta et al. (2006):

a) Ajuste da equação não-linear do diâmetro médio quadrático:

$$
d_{s}=b_{0} \cdot N^{b 1} h_{100}{ }^{b 2}
$$


b) Ajuste da equação não-linear do volume:

$$
V=b_{3} \cdot d_{3}^{b_{6}} \cdot h_{400} \cdot N^{55}
$$

c) Ajuste do nível de densidade utilizando o índice de espaçamento relativo.

$$
N=\frac{20000 \cdot 100^{2}}{\sqrt{3} \cdot R S^{2} \cdot h_{100}{ }^{2}}
$$

d) Representação das isolinhas de diâmetro médio quadrático usando a equação (2), através da geração constante de $\mathrm{d}_{\mathrm{g}}$ e de uma série de $\mathrm{h}_{100}$ :

$$
N=\left(\frac{d_{s}}{b_{0} h_{100}{ }^{62}}\right)^{1 / 61}
$$

e) Representação de isolinhas para o volume, introduzindo na equação (3) as equações (2) e (5), respectivamente, tem-se a solução de $\mathrm{N}$ através de uma série de $\mathrm{h}_{100}$.

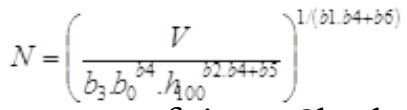

f) Para calcular e representar o coeficiente Slenderness ajustou-se a equação 7 em função do diâmetro:

$$
C S=d /\left(a+b \cdot d+c \cdot d^{2}\right)
$$

Os modelos de regressão foram ajustados no procedimento GLM e NLIN, com o método Gauss-Newton, para regressões lineares e não lineares respectivamente, no pacote SAS - Statistical Analysis System (SAS, 1999).

Os Diagramas de Manejo da Densidade da População foram elaborados em planilhas programadas no Excel, através da sequência de equações anteriormente descrita.

\section{RESULTADOS E DISCUSSÃO}

O índice de espaçamento relativo (RS) expresso em percentagem foi utilizado para caracterizar o nível de crescimento do estoque volumétrico, calculado como a razão entre a distância média entre as árvores e altura dominante. Este índice de espaçamento relativo foi usado porque é particularmente útil para caracterizar os níveis de crescimento do estoque no diagramas de manejo da densidade do povoamento, sendo comumente determinado para controlar a densidade em plantações submetidas a regime de desbaste.

Os dados de índice de espaçamento relativo (RS) originados de parcelas permanentes foram relacionados com a altura dominante e o diâmetro médio, tendo apresentado um coeficiente de determinação igual a 0,5952, erro padrão da estimativa igual a 0,0998 e um coeficiente de variação igual a 3,47 \%, representado pela equação abaixo:

$$
\ln R S=5,16463+0,60208 \cdot \ln d-1,37786 \cdot \ln h_{100}
$$

O valor do Índice de Espaçamento Relativo (RS) de 36\% foi obtido na idade jovem de 5 anos, com altura dominante de $8,5 \mathrm{~m}$, com grande disponibilidade de espaço vital. O valor do Índice de Espaçamento Relativo de 17,1\%, indicando uma situação de início de competição dos indivíduos da população, foi encontrado na idade adulta aos 20 anos, com altura dominante de 21,9 m. Este estágio de competitividade já foi demonstrado para Pinus elliottii por Fishwick (1975), que indicou um índice de $16 \%$ como de elevado nível de competição. Este índice constitui-se num importante indicador para regulação da densidade em povoamentos comerciais, com a aplicação de desbastes seletivos.

O ajuste da equação não-linear do diâmetro médio quadrático, como função do número de árvores por hectare e altura dominante, apresentou um coeficiente de determinação igual a 0,89 , erro padrão da estimativa de 1,104 e um coeficiente de variação igual a 0,78\%, tendo resultado o seguinte modelo:

$$
d_{g}=4,4113 . N^{-0,1072} h_{100}^{0,7953}
$$

Da mesma forma, o ajuste da equação não-linear do volume, como função do número de árvores por hectare e altura dominante, apresentou um coeficiente de determinação igual a 0,99, erro padrão da estimativa de 6,654 e um coeficiente de variação igual a 2,05\%, obtendo-se o modelo:

$$
V=0,000019 \cdot d_{g}{ }^{2,3359} \cdot h_{100}{ }^{0,7641} \cdot N^{1,0189}
$$

Sci. For., Piracicaba, v. 44, n. 110, p. 425-434, jun. 2016 DOI: dx.doi.org/10.18671/scifor.v44n110.15 
O Coeficiente de Conicidade ou Slenderness (CS), foi usado para representar o índice da forma de tronco das árvores e para determinar a suscetibilidade das árvores a danos pela ação do vento, como também foi constatado por Navratil (1995) e Wang et al. (1998). Este índice é uma relação da altura total $(\mathrm{m})$ pelo diâmetro à altura do peito $(\mathrm{cm})$ das árvores, sendo que os valores de CS mais elevados indicam menor estabilidade das árvores e maior vulnerabilidade a danos pela ação do vento.

No caso específico deste estudo, os valores médios do Coeficiente de Conicidade ou Slenderness (CS) foram encontrados para a maioria das árvores com poucos danos pelo vento, bem como, uma amostra de árvores residuais intactas, com valores de CS abaixo da média (Figura 1). Os CS não foram estimados para as árvores que não puderam ser medidas com precisão, por exemplo, devido a troncos e copas que quebraram com o impacto durante a queda das árvores anterior a avaliação, provocada pelo vento dominante. Foi possível gerar uma equação que apresentou um coeficiente de determinação com alto grau de ajustamento igual a 0,9165, um baixo erro padrão da estimativa igual a 1,3101 e um coeficiente de variação igual a 4,79\%, sendo expressa por:

$C S=d /\left(2,75709+0,8988 \cdot d+0,00415 \cdot d^{2}\right)$

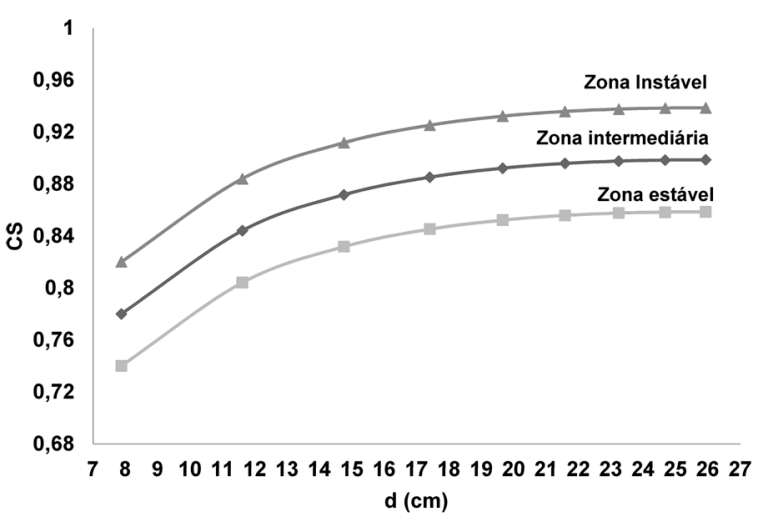

Figura 1. Tendência do Coeficiente de Conicidade ou Slenderness (CS) em função do diâmetro médio.

Figure 1. Trend of the Slenderness coefficient related to mean diameter.

Os valores do Coeficiente de Conicidade ou Slenderness (CS) variaram de 0,78 aos 4 anos, com diâmetro médio à altura do peito de $9,8 \mathrm{~cm}$. Houve estabilização para o valor de CS igual a 0,89 aos 20 anos, com diâmetro médio de $25,9 \mathrm{~cm}$. Considerando que os povoamentos apresentavam pouquíssimos danos pela ação do vento, o que se manifestou em algumas situações onde o CS era superior a 0,9 (90\%), mas em povoamentos com relevo de inclinação acentuada e exposição a ação de ventos dominantes. Na situação dos povoamentos onde se encontravam as parcelas permanentes, que sofreram amostragem de dados, o coeficiente slenderness foi inferior a 0,9 , sem danos acentuados pelo vento, indicando que o regime de manejo da densidade populacional não coloca as árvores em situação de instabilidade à ação do vento, pois os valores de altura média são inferiores aos dos diâmetros das árvores.

De posse da tendência média do CS em função do diâmetro (d) e assumindo o desvio padrão da amostra de 0,04 foi possível traçar a tendência dos limites inferior e superior, subtraindo e somando o valor de um desvio padrão de CS. Isto possibilitou localizar as zonas estável, intermediária e instável para a população de Pinus taeda manejada com desbastes, o que provoca a alteração da densidade populacional. Quando o regime de desbaste realizado for muito pesado, a população passa a situar-se na zona instável, ficando muito suscetível a ação do vento, podendo ocorrer à queda de árvores. Ao contrário, quando o regime de desbaste for leve a moderado a população manejada situa-se na zona estável, onde as árvores sofrem pouco risco pela ação do vento.

Para inserção o coeficiente de conicidade ou slenderness no diagrama de manejo da densidade, inicialmente foi realizado uma alteração na forma de relacionamento das variáveis de crescimento. Neste caso, os diagramas foram construídos com relacionamento do crescimento com índices que representam o tamanho médio da árvore, por exemplo, volume, altura ou diâmetro, com a densidade expressa em número de árvores por hectare. Para isto, vários índices de densidade foram 
utilizados: o índice de densidade de suporte da regra de autodesbaste (TANG et al., 1994), o índice de densidade relativa (DREW; FLEWELLING, 1979), o índice de espaçamento relativo (YODA et al., 1963), e o coeficiente de conicidade ou slenderness (WOON; O'HARA, 2001).

O Diagrama de Manejo da Densidade (DMD) foi elaborado através de uma relação de variáveis dendrométricas, para inclusão do índice de espaçamento relativo e coeficiente de conicidade ou slenderness, para caracterização do estoque de madeira (Figura 2). Desta forma, o Diagrama de Manejo da Densidade fornece informações sobre diâmetro médio, número de árvores, volume total, cruzados com o índice de espaçamento relativo. Além disto, foi incluída uma estimativa do coeficiente slenderness médio para toda a trajetória de densidade e para cada distribuição de diâmetro do povoamento. Esta informação do coeficiente slenderness pode ser usada para avaliar a estabilidade de todo o povoamento e as diferentes classes de copa, respectivamente. O DMD aqui descrito permite aos silvicultores fazer comparações rápidas e fáceis entre as diferentes opções de regimes desbastes, em que, é considerado tanto a produção de madeira quanto o risco de danos causados às árvores pela ação do vento.

O eixo da altura dominante varia de 10 a $36 \mathrm{~m}$, enquanto que as densidades variam 100 a 3200 árvores por hectare. A isolinha superior no diagrama para o índice de espaçamento relativo (RS) corresponde a um valor de $12 \%$ e linhas adicionais de RS variam até $28 \%$. O diagrama também fornece linhas que representam valores constantes de diâmetro médio e volume total. Os valores para o diâmetro quadrático médio variaram de 12 a $44 \mathrm{~cm}$, demonstrado pela inclinação das isolinhas acima, da esquerda para a direita, sendo altamente sensível à densidade. Os valores de volume total do povoamento variaram de 150 a $800 \mathrm{~m}^{3} \mathrm{ha}^{-1} \mathrm{com}$ as isolinhas inclinadas para cima, da esquerda para a direita, de acordo com o princípio de que a produtividade em qualquer ponto no tempo é muito afetada pela altura dominante.

As isolinhas correspondentes a gama de valores de coeficiente slenderness (CS) foram sobrepostas ao DMD (Figura 2) determinada como o limiar de estabilidade neste estudo, tendo definido adequadamente o limite entre as zonas estável, intermediária e instável do povoamento. A consistência destes resultados com os achados por outros autores sugerem que existem zonas gerais de estabilidade para povoamentos homogêneos de coníferas de zonas temperadas.

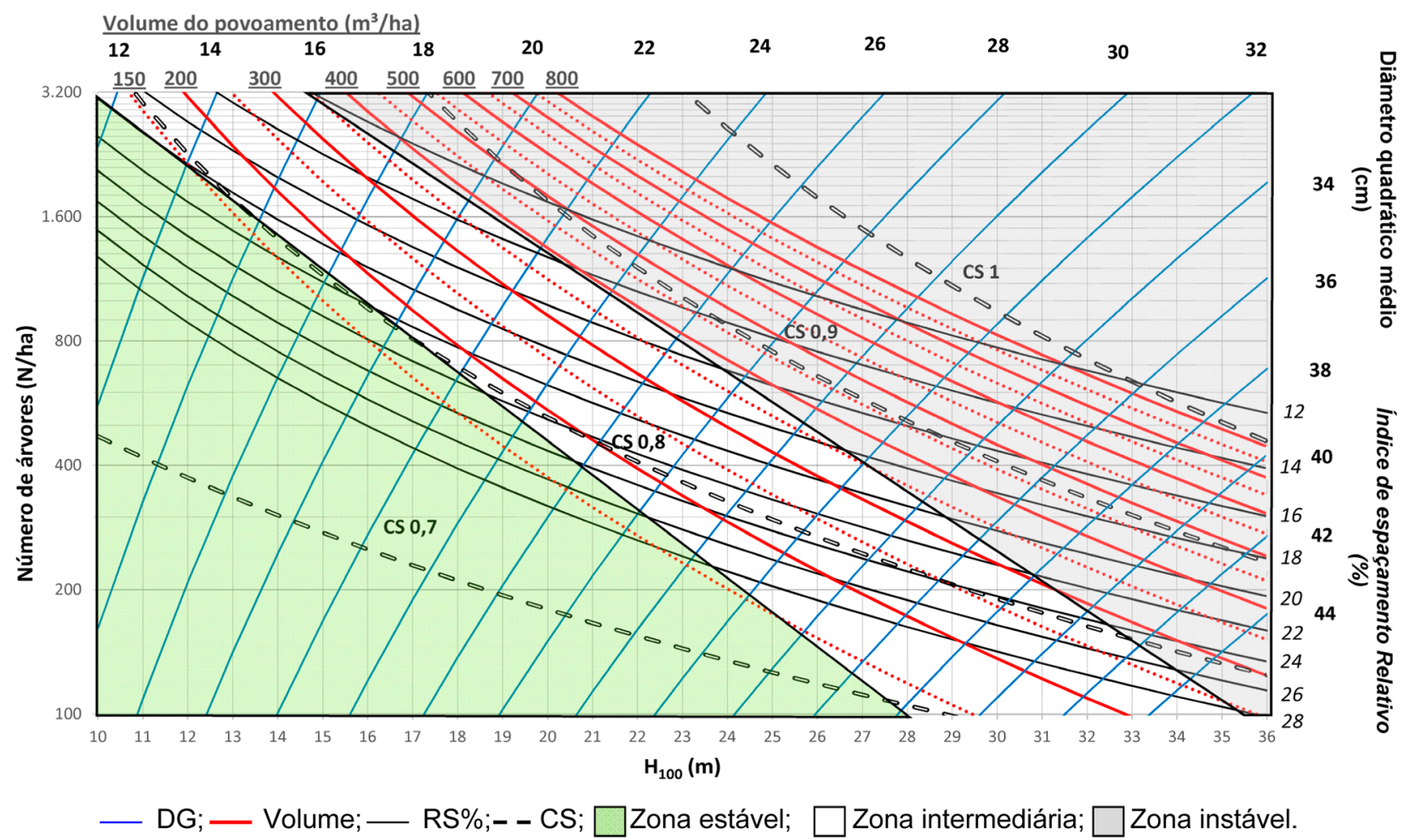

Figura 2. Diagrama de Manejo da Densidade para povoamentos de Pinus taeda incluído as isolinhas dos índices de espaçamento relativo, coeficiente slenderness e as zonas de estabilidade.

Figure 2. Density Management Diagram for Pinus taeda including the iso-lines of the indices of relative spacing, slenderness ratio and the areas of stability. 
Schneider et al. - Utilização do Índice de conicidade no diagrama

de manejo de densidade em povoamentos de Pinus taeda $\mathrm{L}$.

Para exemplificar a utilização prática do DMD gerado, consideremos um povoamento com 1600 árvores por hectare, com altura dominante de 21,0 metros. Com o cruzamento de ambas informações, obtêm-se como resultados o índice de espaçamento relativo igual $12 \%$, diâmetro quadrático médio de aproximadamente $23 \mathrm{~cm}$ e volume total por hectare de $500 \mathrm{~m}^{3}$. Este povoamento possuiria um CS de 0,9, ou seja, localizado na zona instável do DMD.

\section{CONCLUSÕES}

O Diagrama de Manejo da Densidade (DMD) desenvolvido para determinar os momentos de realização dos desbastes para povoamentos de Pinus taeda, levando em consideração a preocupação com a resistência das árvores à queda pela ação do vento, mostrou-se consistente. Permite a estimativa rápida do volume, número de árvores, diâmetro médio e altura dominante, que podem auxiliar os silvicultores para verificar diversos indicadores de manejo sustentável relacionados com o estoque em crescimento.

O coeficiente de conicidade ou slenderness (CS) suporte foi definido em 0,9 (90\%), utilizado para avaliar o risco de dano devido ao vento, foi satisfatoriamente incluído no DMD, definido para avaliar o componente de risco de quebra de árvores do povoamento pelo vento. Porém, deve-se considerar probabilidade de danos em um local por outros fatores ambientais, entre esses os mais importantes são as raízes, tipo do solo, as características topográficas e as condições meteorológicas da região.

\section{REFERÊNCIAS BIBLIOGRÁFICAS}

BAKER, F. S. Principles of silviculture. New York: McGraw Hill, 1950, 414p.

BARRETO, L. S. The clarification of self-thinning and thinning. A simulation approach. Silva Lusitana, Lisboa, v.2, n.2, p. 233-238, 1994.

BARRIO, M.; ÁLVAREZ-GONZÁLEZ, J. G. Development of a stand density management diagram for evenaged pedunculate oak stands and its use in designing thinning schedules. Forestry, v. 78, p. 209-216, 2005.

BARRIO-ANTA, M. et al. An ecoregional model for estimating volume, biomass and carbon pools in maritime pine stands in Galicia (northwestern Spain). Forest Ecology and Management. Amsterdan, v. 223, p. 24-34, 2006.

BECKING, J. H. Einige Gesichtspunkte für die Durchfürung von vergleichenden Durchfostungsversunchen in Gleichaltrigen Beständen. In: Proccedings of the IUFRO Congress, Roma, 1954.

BRUNIG, E. F. Das Risiko der forstlichen Funktionen-planung, dargestellt am Beispiel der Sturmgefahrdung. Allgemeine Forst-und Jagd-Zeitung, Berliner, n. 145, p. 60-67, april, 1974.

BURKHART, H. E. Estimating growth and mortality. Virginia: Virginia Polytechnic Institute and State University, 1974. 15p.

CASTEDO-DORADO, F. et al. Daños producidos por la nieve en masas de Pinus radiata D. Don en zonas de media montaña lucense. Recomendaciones selvícolas, Congreso de Ordenación y Gestión Sostenible de Montes. Santiago de Compostela (Spain), p. 457-465. 2000.

CASTEDO-DORADO, F.; CRECENTE-CAMPO, F.; ÁLVAREZ-ÁLVAREZ, P.; BARRIO-ANTA, M. Development of a stand density management diagram for radiata pine stands including assessment of stand stability. Forestry, Oxford, v. 82, n.1, p. 1-16, January, 2009.

CREMER, K. W. et al. Effects of stocking and thinning on wind damage in plantations. New Zealand Journal of Forestry Science, Heidelberg, n. 12, v. 2, p. 244-268, 1982. 
DAVIS, K. P. Forest Management: Regulation and Valuation. 2 ed. McGraw-Hill, New York, 519 p., 1966.

DEAN, J. T.; BALDWIN, V. C. Using a density-management diagram to develop thinning schedules for loblolly pine plantations. USDA Forest Service, Southern Forest Experimental Station. Res. Pap., SO 275. 1993.

DEAN, T. J.; BALDWIN, V. C. Crown management and stand density. In: Carter, Mason (Eds.), Growing Tress in a Greener World: Industrial Forestry in the 21st Century, 35th LSU Forestry Symposium. Louisiana State University Agricultural Center, Louisiana Agricultural Experiment Station, Baton Rouge, LA, p.148-159. 1996.

DEL RIO, M.; MONTERO, G.; BRAVO, F. Analysis of diameter-density relationships and self-thinning in nonthinned even-aged Scots pine stands. Forest Ecology and Management. Amsterdan, v.142, p.79-87, March, 2001.

DREW, T. J.; FLEWELLING, J. W. Stand density management: an alternative approach and its application to Douglas-fir plantations. Forest Science. Bethesda, n.25, p. 518-532, September, 1979.

EFTHIMIOU, G. The slenderness of the softwood Riparian forest species Salix alba L. and Salix fragilis L. in the protected area of Nestos Delta, Greece. Journal of Ecology and the Natural Environment, n. 4, v. 1, p. 1-7, January, 2012.

EMBRAPA. Sistema brasileiro de classificação de solos. Brasília: Embrapa, 1999, 412 p.

FABER, P. J. Stability of stands to wind: a theoretical approach. Nederlands Bosbauw Tijdschrift, n. 47, v. 7/8. p. 179-193, 1975.

FISHWICK, R. W. Uso do percentual de espaçamento relativo de Hart-Becking para o controle dos desbastes. Brasília: PRODEPEF/IBDF, 1975, 7p.

HINZE, W. H. F; WESSELS, M. O. Stand stability in pines: an important silvicultural criterion for the evaluation of thinnings and the development of thinning regimes: management paper. South African Forestry Journal, Grahamstown, n. 196, p. 37-40, 2002.

JACK, S. B.; LONG, J. N. Linkages between silviculture and ecology: analysis of density management diagrams. Forest Ecology and Management. Amsterdan, v. 86, n. 1-3, p. 205-220, 1996.

LEE, Y. L. Predicting mortality for even-aged stands of lodgepole pine. The Forestry Chronicle, Ottawa, v. 47, n.1, p. 29-32, 1971.

LOHMANDER, P.; HELLES, F. Windthrow probability as a function of stand characteristics and shelter. Scandinavian Journal of Forest Research, Helsinki, v. 1-4, n. 2, p. 227-238, 1987.

MOTA, F. S.; BEIRSDORF, M. J. C.; GARCEZ, R. B. Zoneamento agrícola do Rio Grande do Sul e Santa Catarina: normas agro-climáticas. Pelotas: Ministério da Agricultura. 1971, 80p.

NAVRATIL, S. Minimizing damage in alternative silviculture systems in boreal mixed woods. Can. For. Serv. North. For. Cent./Alberta Land For. Serv., Edmonton, AB. Rep. Fo42-91/124-1995E. 1995. 74 p.

NEWTON, P. F. Stand density management diagrams: review of their development and utility in stand-level management planning. Forest Ecology and Management. Amsterdan, v.98, p.251-265. March, 1997.

NEWTON, P. F; WEETMAN, G. F. Stand density management diagram for managed black spruce stands. The Forestry Chronicle, Ottawa. v. 70, n. 1, p. 65-74. 1994. 
Schneider et al. - Utilização do Índice de conicidade no diagrama

de manejo de densidade em povoamentos de Pinus taeda $\mathrm{L}$.

NYKÄNEN, M. L. et al. Factors affecting snow damage of trees with particular reference to European conditions. Silva Fennica, Helsinki, n. 31, v. 2, p. 193-213, 1997.

PARDÉ, J.; BOUCHON, J. Dendrométrie 2ème edn. Nancy, France: Ecole Nationale du Génie Rural, des Eaux Et des Forêts, 1988.

RICE, J. A.; MAN, R. Wind damage in a partially harvested boreal mixedwood stand in northeastern Ontario. Ontario Forest Research Institute, Forest Research Report, n. 175, 14 p., 2011.

SAS Institute Inc. SAS/STAT user's guide. Version 8 (computer manual). SAS Institute Inc., Cary. N.C. 1999.

SMITH, N. J.; HANN, D. W. A growth model based on the self-thinning rule. Canadian Journal of Forest Research. New Westminster, v. 16, n. 2, p. 330-334, April, 1986.

TANG, S.; MENG, C. H.; MENG, F. R.; WANG, Y. H. A growth and self-thinning model for pure even-aged stands: theory and applications. Forest Ecology and Management. Amsterdan, v. 70, n. 1-3, p, 67-73, December, 1994.

WANG, Y.; TITUS, S. J.; LEMAY, V. M. Relationships between tree slenderness coefficients and tree or stand characteristics for major species in boreal mixedwood forests. Canadian Journal of Forest Research. New Westminster, n. 28, v. 8, p. 1171-1183, 1998.

WESTOBY, M. The place of the self-thinning rule in population dynamics. The American Naturalist. The University of Chicago Press, v.118, n.4, p. 581-587, 1981.

WONN. H. T.; O'HARA, K. L. Height:Diameter Ratios and Stability Relationships for Four Northern Rocky Mountain Tree Species. Western Journal of Applied Forestry, Society of American Foresters, n. 16, v. 2. p. 87-94, April, 2001.

YODA, K. et al. Self-thinning in over crowed pure stands under cultivated and natural conditions. Journal Biology, Osaka University, Osaka, v.14, p.107-129, 1963.

ZEIDE, B. Analysis of the 3/2 power law of self-thinning. Forest Science. Bethesda, v.33, n.2, p. 17-537, June, 1987.

ZEIDE, B. Self-thinning and stand density. Forest Science. Bethesda, v.37, n.2, p. 517-523, June, 1991.

Recebido em 20/11/2014

Aceito para publicação em 30/11/2015 